\title{
Super-resolution Microscopy with Structured Excitation and Structured Stimulated Emission Depletion
}

\author{
Qingru Li, Jiang Zhang, Hua Huang, Ming Tang, Xiyue Wang and Han Zhang* \\ School of Electrical Engineering and Information, Sichuan University, Chengdu, China \\ ${ }^{*}$ Corresponding author
}

\begin{abstract}
Fluorescence microscopy plays an important role in biomedical imaging because of its high sensitivity and specificity. However, the resolution of traditional fluorescence microscopy is limited due to the optical diffraction. Various techniques have been developed to surpass the diffraction limit in recent years. Among these existing methods, nonlinear structured illumination microscopy (SIM) simultaneously provides the ability of fast imaging speed, wide field of view and extended resolution improvement. However, the current developed nonlinear SIM approaches such as Saturated SIM and Photoswitching SIM have their own defects due to the strong photon bleaching and slow photoswitching speed respectively. We report a new nonlinear SIM technique based on stimulated emission depletion (STED), the illumination pattern of which combining both structured excitation field and structured STED field (SSTED-SIM). Theoretical study and simulation analysis have been conducted to demonstrate that SSTED-SIM performs better than other existing nonlinear SIM.
\end{abstract}

Keywords-resolution; fluorescence microscopy; biological imaging; image processing

\section{INTRODUCTION}

Fluorescence microscopy plays a key role in the study of cellular structure and function, because of its high specificity, great sensitivity and fast imaging speed. However, its optical resolution is limited to the Full Width at Half Maximum (FWHM) of Point Spread Function (PSF) owing to the diffraction limit, which greatly restricts the observation of biological samples. Recently, there are various super-resolution fluorescence microcopy methods which can break the diffraction limit [1-7], and those methods can be categorized into two groups. The first kind is super-resolution fluorescence microcopy based on the illumination pattern narrowing the PSF, such as stimulated emission depletion (STED) microscopy $[8,9]$ reversible saturable optical fluorescence transitions (RESOLFT) [10] and structured illumination microscopy (SIM) [11]. The other is based on precise positioning of single molecules by switching on and off fluorophores randomly, including photoactivated localization microscopy (PALM \& fPALM) [12, 13], stochastic optical reconstruction microscopy (STORM) [14], and ground-state depletion with individual molecule return microscopy (GSDIM) [15]. Yet these super-resolution methods have their respective advantages and disadvantages. PALM, STORM and GSDIM have high resolution and sensitivity but limited speed due to requiring hundreds of raw images in reconstruction. STED has the ability to achieve rapid imaging, yet as a point-scanning method, laser-scanning STED has a compromise between imaging speed and field of view. Synthetically, SIM is the most suitable method for live cell imaging, but SIM can only improve resolution by a factor of two [11].

However, nonlinear structured illumination microscopy (NSIM) based on nonlinear characteristics of fluorophores is capable of significantly enhancing imaging resolution. Some nonlinear effects have already been used in the nonlinear fullfield super-resolution imaging study [16-21]. Saturated structured illumination microscopy (SSIM) improves the resolution to $50 \mathrm{~nm}$, but it is not suitable for live cell study because of its severe photobleaching [16]. Photoswitching SIM utilizing the nonlinear effect of photoswitchable fluorophores is inappropriate to live cell imaging either, since the long photoswitching time limits the imaging speed [18]. Applying STED as the nonlinear effect in NSIM has been previously proposed. In the study of standing-wave total internal reflection microscopy (SW-TIRM), by adding a standing-wave STED field to a standing-wave excitation field, it can promote the resolution of SW-TIRM theoretically [19]. Theoretical study of STED-SIM with non-structured excitation light and structured STED light has been proposed already, and simulation analysis predicted that 2D-STED effect enhanced by surface plasmon resonance (SPR) can achieve high-speed imaging at 30-nm resolution in nonlinear SIM [20]. And nonlinear SIM based on structured excitation light and structured STED light (SSTEDSIM) was also suggested but without in-depth analysis [21]. In this paper, we report an approach that applies nonlinear structured illumination by combining both patterned excitation field and patterned stimulated emission depletion (STED) field, which termed as SSTED-SIM. Profound theoretical study and simulation analysis of SSTED-SIM are performed. As STED effect's rapid switching response, insignificant shot noise and theoretical unlimited resolution, SSTED-SIM is supposed to be a better nonlinear SIM than any existing nonlinear SIM. And the result shows that SSTED-SIM has a narrower point spread function and greater ability of solving high-frequency harmonics compared to STED-SIM, which indicates that SSTED-SIM has high technical feasibility and practical operability.

\section{THEORY}

The algorithm of linear SIM super resolution imaging reconstruction process is presented firstly to help understand the proposed SSTED-SIM image reconstruction theory. By applying a linear patterned illumination on the object, the illuminated object can be depicted as the multiplication of the 
object and the illumination pattern in spatial domain, as described in (1). By convolving the illuminated object with the point spread function (PSF) of the imaging system, the ultimate observed image will be constructed, as described in (2).

$$
\begin{gathered}
\mathrm{IO}(\mathrm{x})=\mathrm{O}(\mathrm{x}) \times \text { Illumination }(\mathrm{x}) . \\
\mathrm{IM}(\mathrm{x})=[\mathrm{O}(\mathrm{x}) \times \text { Illumination }(\mathrm{x})] \otimes \mathrm{PSF}(\mathrm{x}) .
\end{gathered}
$$

Where $\mathrm{O}$ is the object, Illumination, PSF, IO and IM are the illumination pattern, point spread function, illuminated object and ultimate observed image respectively.

The image forming process in spatial frequency domain is shown in (3)- (4): the ultimate observed image in frequency domain is the result of the object convolving with the illumination pattern in frequency domain and then multiplied by optical transfer function (OTF) of the imaging system.

$$
\begin{gathered}
\mathrm{IO}(\xi)=\mathrm{O}(\xi) \otimes \text { Illumination }(\xi) . \\
\mathrm{IM}(\xi)=[\mathrm{O}(\xi) \otimes \text { Illumination }(\xi)] \times \mathrm{OTF}(\xi) .
\end{gathered}
$$

Where $\xi$ is the spatial frequency of the illumination pattern.

For linear SIM, the illumination pattern is expressed as in (5). The Fourier transform of (5) is shown by (6), which is composed by three shifted $\delta$ functions [22], where $\mathrm{c}_{\mathrm{m}}$ is the strength of $\mathrm{m}_{\text {th }}$ order harmonic. Equation (7) depicts the Fourier domain expression of (4) and contains a non-shifted and two shifted object information in frequency domain. Equation (8) and (9) depict multiple images obtained by using different illumination pattern with different phases. Super-resolution image which is capable of surpassing the diffraction can be reconstructed by solving IO $(\xi)$, IO $\left(\xi+\mathrm{k}_{0}\right), \mathrm{IO}\left(\xi-\mathrm{k}_{0}\right)$ in (7)- (9), where $\mathrm{k}_{0}$ is the spatial frequency of the pattern period.

$$
\begin{gathered}
\text { Illumination }(\mathrm{x})=1+\cos \left(2 \pi \mathrm{k}_{0} \mathrm{x}+\varphi\right) \\
\text { Illumination }(\xi)=\sum_{\mathrm{m}=-1}^{1} c_{m} \delta\left(\xi+m k_{0}\right) e^{-i m \varphi} \\
\mathrm{IM}_{1}(\xi)=\sum_{m=-1}^{1} c_{m} \operatorname{IO}\left(\xi+m k_{0}\right) e^{-i m \varphi} \times \mathrm{OTF}(\xi) . \\
\mathrm{IM}_{2}(\xi)=\sum_{m=-1}^{1} c_{m} \operatorname{IO}\left(\xi+m k_{0}\right) e^{-i m \varphi_{1}} \times \mathrm{OTF}(\xi) . \\
\operatorname{IM}_{3}(\xi)=\sum_{m=-1}^{1} c_{m} \operatorname{IO}\left(\xi+m k_{0}\right) e^{-i m \varphi_{2}} \times \mathrm{OTF}(\xi) .
\end{gathered}
$$

Building on previous research $[20,21], \pi$ is the best phase difference between the excitation grid and the STED grid for 2DSSTED-SIM to obtain maximal nonlinearity caused by STED effect. The illumination pattern of SSTED-SIM can be written as

$$
\mathrm{I}(\mathrm{x})=\frac{I_{e x}\left[2+\cos \left(2 \pi \mathrm{k}_{0} x+\varphi_{x}+\pi\right)+\cos \left(2 \pi \mathrm{k}_{0} y+\varphi_{y}+\pi\right)\right]}{1+I_{\text {STED }}\left[2+\cos \left(2 \pi \mathrm{k}_{0} x+\varphi_{x}\right)+\cos \left(2 \pi \mathrm{k}_{0} y+\varphi_{y}\right)\right]}
$$

Where $I(x)$ is the two-structured illumination, Iex is the excitation power, ISTED is the intensity distribution of STED light, $\varphi_{\mathrm{x}}$ and $\varphi_{\mathrm{y}}$ are the phases of 'the two-dimensional illumination pattern. The effective illumination pattern of SSTED is simulated and shown in Figure 1.

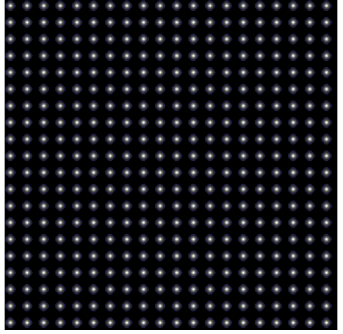

FIGURE I. EFFECTIVE NONLINEAR ILLUMINATION PATTERN OF SSTED-SIM

To reconstruct a super-resolution image, a set of raw images are obtained with different pattern phases from 0 to $2 \pi$ shifted in $\mathrm{N} \times \mathrm{N}$ steps along two directions. One of the $\mathrm{N} \times \mathrm{N}$ raw pictures under a grid phase $\left(\varphi_{\mathrm{x}}=2 \mathrm{p} \pi / \mathrm{N} ; \quad \varphi_{\mathrm{y}}=2 \mathrm{q} \pi / \mathrm{N}\right)$ should be

$$
\begin{aligned}
& P(x, y, p, q)=O(\mathrm{x}, y) \\
& \times \frac{I_{e x}\left[2+\cos \left(2 \pi \mathrm{k}_{0} x+\frac{2 p \pi}{N}+\pi\right)+\cos \left(2 \pi \mathrm{k}_{0} y+\frac{2 q \pi}{N}+\pi\right)\right]}{1+I_{S T E D}\left[2+\cos \left(2 \pi \mathrm{k}_{0} x+\frac{2 p \pi}{N}\right)+\cos \left(2 \pi \mathrm{k}_{0} y+\frac{2 q \pi}{N}\right)\right]} \\
& \otimes P S F(x, y)
\end{aligned}
$$

Where $\mathrm{p}, \mathrm{q}$ are integers between 1 and $\mathrm{N}, \mathrm{O}(\mathrm{x}, \mathrm{y})$ is the object, PSF $(\mathrm{x}, \mathrm{y})$ is the point spread function of the imaging system. The Fourier transform of raw image $\mathrm{P}$ can be written as

$$
\begin{aligned}
& P\left(k_{x}, k_{y}, p, q\right)=\sum_{m, n=-N_{i}}^{N_{i}} C_{m n} I O\left(k_{x}+m k_{0}, k_{y}+n k_{0}\right) \\
& \times O T F\left(k_{x}, k_{y}\right)
\end{aligned}
$$

Where $\mathrm{Ni}$ is the harmonic order to be solved, and $\mathrm{C}_{\mathrm{mn}}$ is the strength of the Fourier frequency component, IO $\left(\mathrm{k}_{\mathrm{x}}+\mathrm{mk}_{0}\right.$, $\left.\mathrm{k}_{\mathrm{y}}+\mathrm{nk}_{0}\right)$ is the frequency-shifted illuminated object image IO (x, y) in Fourier transform. By solving $\mathrm{N} \times \mathrm{N}$ equations like (12), IO $\left(\mathrm{k}_{\mathrm{x}}+\mathrm{mk}_{0}, \mathrm{k}_{\mathrm{y}}+\mathrm{nk}_{0}\right)$ can be reconstructed. Then a high resolution Fourier image would be obtained with the appropriate combination of all IO $\left(\mathrm{k}_{\mathrm{x}}, \mathrm{k}_{\mathrm{y}}\right)$ components. The final superresolution image is formed through means of inverse fast Fourier transform (IFFT) algorithm. As shown in Figure 2, the entire process of image reconstruction was simulated. 

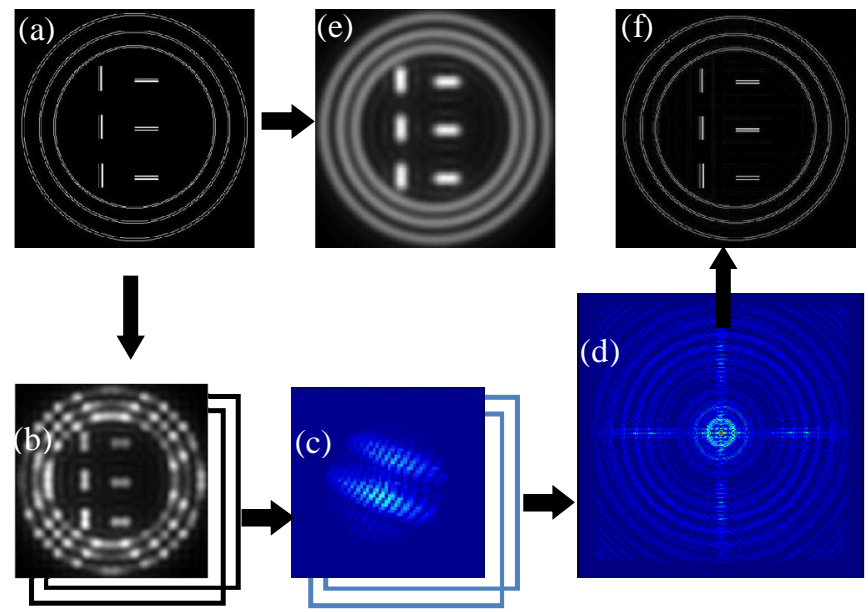

(d)

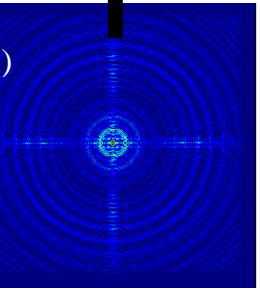

FIGURE II. (A) SIMULATED OBJECT, WHICH CONTAINS THREE PAIRS OF CIRCLES SEPARATED BY 20,30, 40NM RESPECTIVELY, THREE PAIRS OF HORIZONTAL BARS AND VERTICAL BARS SPACING AT 20, 30, 40NM RESPECTIVELY. (B) SIMULATED RAW IMAGES AFTER EXCITED BY STRUCTURED ILLUMINATION WITH VARIED PHASES. (C) FOURIER COMPONENTS IN DIFFERENT FREQUENCY DOMAIN AFTER IMAGE PROCESSING. (D) RECONSTRUCTED IMAGE IN FREQUENCY DOMAIN. (E) SUPER-RESOLUTION IMAGE IN SPATIAL DOMAIN. (F) SIMULATED DIFFRACTION LIMIT IMAGE FROM OBJECT IN (A).

\section{DISCUSSION}

There are several nonlinear SIM approaches which have been promoted previously. In Saturated-SIM [16], the nonlinear relationship between fluorescence distribution and excitation strength is given by

$$
\text { Fluorescence } \sim \frac{I_{e x}}{I_{e x}+I_{s a t}}
$$

Where $I_{e x}$ is the power of the excitation light and $I_{\text {sat }}$ is a constant representing the saturation power of the fluorophore. The saturated structured illumination pattern induced from sinusoidal lights with different maximum is shown in Figure 3 (a). As the excitation grows compared with saturation power, the fluorescence intensity would peak at certain position. The saturated structured-illumination turns most part of the sample into the 'on' state. In comparison, the fluorescence distribution in STED-SIM follows the nonlinear relationship [23]:

$$
\text { Fluorescence } \sim \frac{1}{I_{S T E D} / I_{\text {sat }}+1}
$$

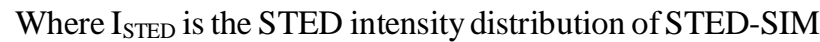
illumination pattern. As described in Figure 3 (b), the effective illumination pattern always presents a sinusoidal shape with different intensity of STED light. This can guarantee a better quality of super-resolution image in STED-SIM.

By comparison, the nonlinear relationship between fluorescence intensity and SSTED-SIM illumination follows:

$$
\text { Fluorescence } \sim \frac{I_{e x}}{I_{S T E D} / I_{s a t}+1}
$$

It is expected that the effective illumination of SSTED-SIM occurs in a sinusoidal shape as well as STED-SIM. Through careful comparison, we can tell that the effective illumination's full width at half maximum (FWHM) of SSTED-SIM is lower than STED-SIM at the same excitation, especially when the excitation power is weak. Which indicates SSTED-SIM can conduct high quality super-resolution imaging at a weak excitation power.

(a)

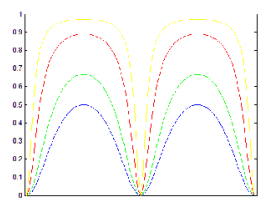

FIGURE III. FLUORESCENCE DISTRIBUTION IN (A) SATURATED SIM (B) STED-SIM (C) SSTED-SIM; DIFFERENT COLORS OF THE CURVES REPRESENT DIFFERENT EFFECTIVE ILLUMINATION PATTERNS WITH VARIED TIMES OF ISAT THE BLUE, GREEN, RED, YELLOW STAND FOR 0.5, 1, 4, 16 TIMES OF ISAT RESPECTIVELY.

The strength of nonlinear-SIM's Fourier components changes continuously with excitation power increasing. As for Saturated SIM, it is difficult to solve high-frequency harmonics because of its self-limiting feature, which can be proved by Figure 4. Different colors of the curves represent different harmonics orders. The dashed line represents hypothetical lowest detectable SNR level in imaging processing. The harmonics orders above the dashed line can be retrieved after reconstruction. The strength of harmonics decreases with the increase of excitation power, which leads to the self-limited resolution feature of Saturated SIM.

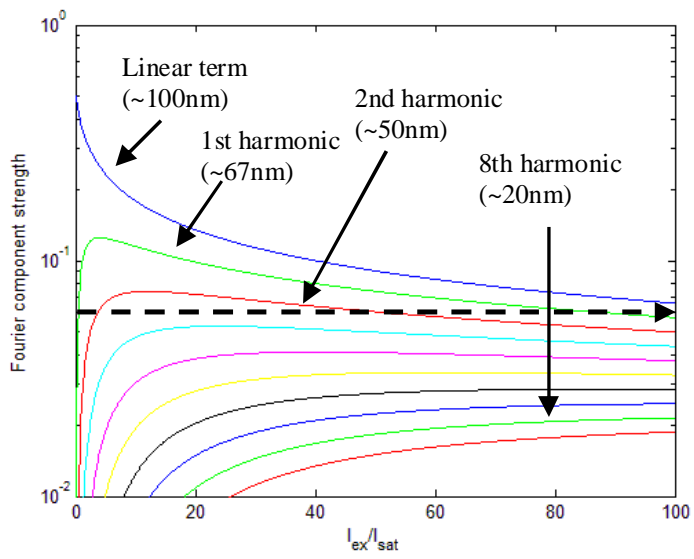

FIGURE IV. THE STRENGTH OF SATURATED-SIM FOURIER COMPONENTS AS EXCITATION POWER INCREASES. DIFFERENT COLORS OF THE CURVES INDICATE DIFFERENT HARMONICS ORDERS: BLUE REPRESENTS LINEAR TERM, GREEN REPRESENTS 1ST HARMONICS ORDER, RED

REPRESENTS 2ND HARMONICS ORDERS AND SO ON. THE DASHED LINE REPRESENTS HYPOTHETICAL LOWEST DETECTABLE SNR LEVEL IN IMAGING PROCESSING. 
Theoretically, the resolution of STED-SIM and SSTED-SIM are unlimited. The Fourier components strength of STED-SIM and SSTED-SIM are described in Figure 5 and Figure 6 respectively. The solvable harmonics orders would increase as the STED power raise. Moreover, Figure 7 clearly shows that the SSTED-SIM is easier than STED-SIM to solve highfrequency harmonics orders under the same signal noise ratio (SNR) level. This points out that the imaging requirements of SSTED-SIM are easier to be satisfied in further experiment and makes SSTED-SIM a better nonlinear SIM in practical application.

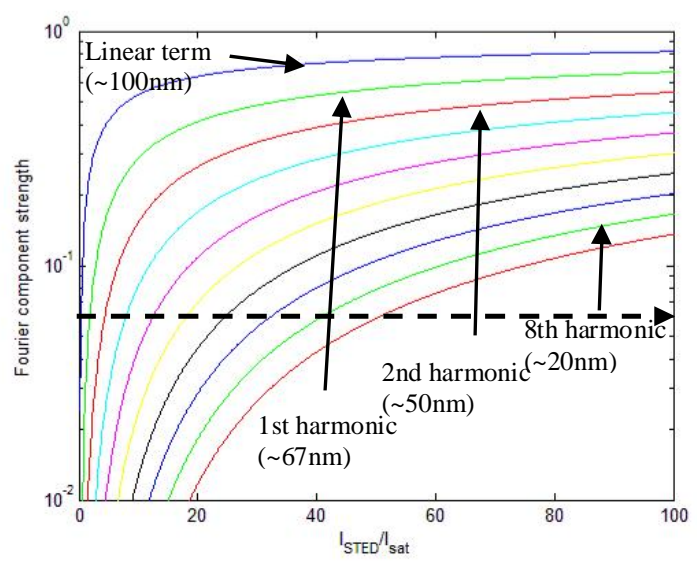

FIGURE V. THE STRENGTH OF STED-SIM FOURIER COMPONENTS AS EXCITATION POWER INCREASES. DIFFERENT COLORS OF THE CURVES INDICATE DIFFERENT HARMONICS ORDERS: BLUE REPRESENTS LINEAR TERM, GREEN REPRESENTS 1ST HARMONICS ORDER, RED REPRESENTS 2ND HARMONICS ORDERS AND SO ON. THE DASHED LINE REPRESENTS HYPOTHETICAL LOWEST DETECTABLE SNR LEVEL IN IMAGING PROCESSING

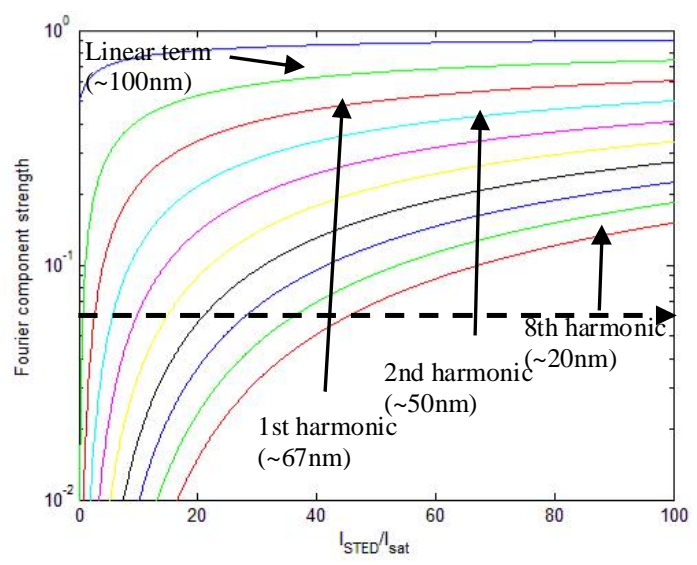

FIGURE VI. THE STRENGTH OF SSTED-SIM FOURIER COMPONENTS AS EXCITATION POWER INCREASES. DIFFERENT COLORS OF THE CURVES INDICATE DIFFERENT HARMONICS ORDERS: BLUE REPRESENTS LINEAR TERM, GREEN REPRESENTS 1ST HARMONICS ORDER, RED

REPRESENTS 2ND HARMONICS ORDERS AND SO ON. THE DASHED LINE REPRESENTS HYPOTHETICAL LOWEST DETECTABLE SNR LEVEL IN IMAGING PROCESSING.

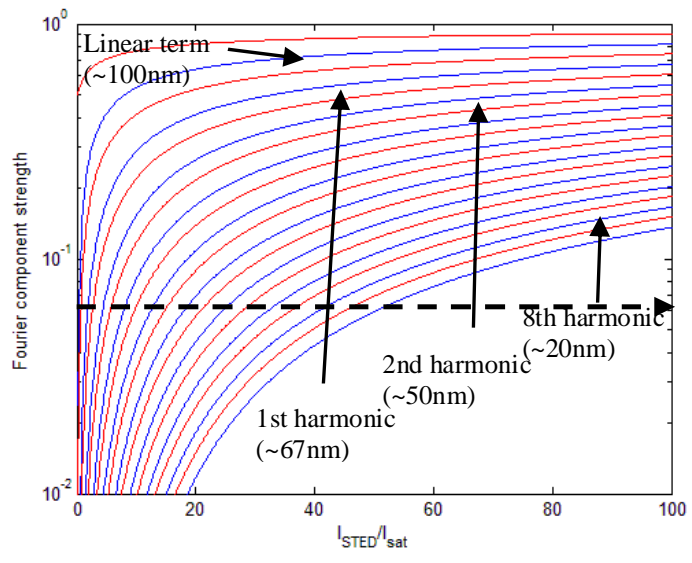

FIGURE VII. THE COMPARISON OF DIFFERENT HARMONICS STRENGTH BETWEEN SSTED-SIM AND STED-SIM: RED FOR SSTED-SIM, BLUE FOR STED-SIM. THE DASHED LINE REPRESENTS HYPOTHETICAL LOWEST DETECTABLE SNR LEVEL IN IMAGING PROCESSING.

\section{SUMMARY}

Based on our previous study, SSTED-SIM technique can provide biomedical imaging with better resolution while applying less STED power. We theoretically demonstrated that the SSTED-SIM provides a good comprehensive performance nonlinear SIM method compared with various existing nonlinear SIM because of its fast imaging speed, low shot noise, great antinoise ability and high feasibility. Simulation results prove that the SSTED-SIM is a potential better super-resolution imaging method with great effectiveness and accountability. This method could make fluorescence microscopy play a better role as it has great potential for biomedical research and application. Further study is under investigation.

\section{ACKNOWLEDGMENT}

This research was financially supported by the Sichuan University scientific research fund.

\section{REFERENCES}

[1] S. W. Hell, "Microscopy and its focal switch," Nat. Methods, vol. 6, pp. 24-32, December 2008.

[2] S. W. Hell, "Far-field optical nanoscopy," Science, vol. 316, pp. 1153 1158, nay 2007.

[3] B. Huang, H. Babcock, and X. Zhuang, "Breaking the diffraction barrier: super-resolution imaging of cells," Cell, vol. 143, pp. 1047-1058 December 2010.

[4] L. Schermelleh, R. Heintzmann, and H. Leonhardt, "A guide to superresolution fluorescence microscopy," Journal of Cell Biology, vol. 190, pp. 165-175, July 2010.

[5] J. Lippincott-Schwartz, and S. Manley, "Putting super-resolution fluorescence microscopy to work," Nature Methods, vol. 6, pp. 21-23., Decemember 2008

[6] B. O. Leung, and K. C. Chou. "Review of super-resolution fluorescence microscopy for biology," Applied Spectroscopy, vol. 65, pp. 967-980, September 2011.

[7] B. Huang,"Super-resolution optical microscopy: multiple choices," Current Opinion in Chemical Biology, vol. 14, pp. 10-14, February 2010.

[8] S. W. Hell and J. Wichmann, "Breaking the diffraction resolution limit by stimulated emission: stimulated emission-depletion fluorescence microscopy,” Opt. Lett, vol. 19, pp. 780-782, June 1994. 
[9] T. Klar, S. Jakobs, M. Dyba, A. Egner, and S. W. Hell, "Fluorescence microscopy with diffraction resolution barrier broken by stimulated emission,” Proc. Natl. Acad. Sci. U.S.A., vol. 97, pp. 8206-10, July 2000.

[10] M. Hofmann, C. Eggeling, S. Jakobs, and S. W. Hell, "Breaking the diffraction barrier in fluorescence microscopy at low light intensities by using reversibly photoswitchable proteins," Proc. Natl. Acad. Sci. U.S.A, vol. 102, pp. 17565-17569, December 2005.

[11] M. G. L. Gustafsson, "Surpassing the lateral resolution limit by a factor of two using structured illumination microscopy," J. Microsc, vol. 198, pp. 82-87, May 2000.

[12] Betzig, Eric, et al. "Imaging Intracellular Fluorescent Proteins at Nanometer Resolution," Science, vol. 313, pp. 1642-1645, September 2006

[13] S. T. Hess, T. P. K. Girirajan, and M. D. Mason, "Ultra-high resolution imaging by fluorescence photoactivation localization microscopy," Biophys. J, vol. 91, pp. 4258-4272, December 2006.

[14] M. J. Rust, M. Bates, and X. Zhuang, "Sub-diffraction-limit imaging by stochastic optical reconstruction microscopy (STORM)," Nat. Methods, vol. 3, pp. 793-796, October 2006.

[15] J. Fölling, M. Bossi, H. Bock, R. Medda, C. A. Wurm, B. Hein, S. Jakobs, C. Eggeling, and S. W. Hell," Fluorescence nanoscopy by ground-state depletion and single-molecule return," Nat. Methods, vol. 5, pp. 943-945, September 2008

[16] M. G. Gustafsson, "Nonlinear structured-illumination microscopy: widefield fluorescence imaging with theoretically unlimited resolution," Proc. Natl. Acad. Sci. U.S.A., vol. 102, pp. 13081-13086, July 2005.

[17] P. Kner, B. B. Chhun, E. R. Griffis, L. Winoto, and M. G. L. Gustafsson, "Super-resolution video microscopy of live cells by structured illumination," Nat. Methods, vol. 6, pp. 339-342, May 2009

[18] E. H. Rego, et al, "Nonlinear structured-illumination microscopy with a photoswitchable protein reveals cellular structures at 50-nm resolution," Proc. Natl. Acad. Sci. U.S.A, vol. 109, pp. E135-E143, May 2011.

[19] C. Y. Dong, H. S. Kwon, and P. T. C. So, "Resolution enhancement in standing-wave total internal reflection microscopy: a point-spreadfunction engineering approach," Journal of the Optical Society of America A Optics Image Science \& Vision, vol. 18, pp. 2833-45, November 2001.

[20] H. Zhang, M. Zhao, and L. Peng, "Nonlinear structured illumination microscopy by surface plasmon enhanced stimulated emission depletion," Optics Express, vol. 19, pp. 24783-94, November 2011.

[21] F. Dake, S. Nakayama, and Y. Taki, "Theoretical assessment of twodimensional nonlinear structured illumination microscopy based on structured excitation and structured stimulated emission depletion." Optical Review, vol. 22, pp. 598-604, August 2015.

[22] B. J. Chang, Chou, L. J., Chang, Y. C., and S. Y. Chiang, "Isotropic image in structured illumination microscopy patterned with a spatial light modulator. " Optics Express, vol. 17, pp. 14710-14721, August 2009.

[23] K. I. Willig, B. Harke, R. Medda, and S. W. Hell, "STED microscopy with continuous wave beams," Nat.Methods, vol. 4, pp. 915-918, November 2007. 